\title{
The Astronomy Education Review: Research, Resources, News, and Opinions
}

\author{
Sidney C. Wolff \\ National Optical Astronomy Observatory, Tucson AZ USA \\ E-mail: swolff@noao.edu
}

The Astronomy Education Review (http://aer.noao.edu), a new electronic refereed journal, began publication in January, 2002. This peer-reviewed journal, which describes itself as "a lively electronic compendium of research, news, resources, and opinion" is the first in the US to be devoted to education and public outreach in astronomy and space science. The journal has been endorsed by both the American Astronomical Society and the Astronomical Society of the Pacific and has received financial support from NASA and NSF. Papers published to date cover a range of topics, and a sample list of subjects includes: 1) the effects of different assessment techniques on student learning; 2) student misconceptions in cosmology and astrobiology: 3) the development, validation, and application of the Astronomy Diagnostic Test; 4) experience with collaborative learning groups in the classroom; 5) implementation and evaluation of a distance learning course for secondary school teachers; 6 ) resource lists for incorporating poetry and science fiction into astronomy classes; and 7) research into the effectiveness of CD-ROMs in the K-12 classroom. We have also published reviews, opinion pieces, and a variety of suggestions for classroom activities.

The overall goal of the journal is to provide a mechanism for communicating information; building collective knowledge; validating the quality of work; and recognizing and rewarding achievement. During the school year, the journal receives an average of 150,000 hits from 5500 unique IP addresses each month. There are no charges to either authors or readers, and to keep costs low, the journal will continue to be available electronically only. The papers published, however, can easily be printed in PDF format. Submissions from countries other than the US are welcome. 